OPEN ACCESS

Edited by:

Jorge L. Alió Del Barrio, Miguel Hernández University of

Elche, Spain

Reviewed by:

José F. Alfonso,

Fernández-Vega Ophthalmological

Institute, Spain

Daniel Elies,

Instituto de Microcirugía Ocular, Spain

${ }^{*}$ Correspondence:

Kazutaka Kamiya

kamiyak-tky@umin.ac.jp

Specialty section: This article was submitted to

Ophthalmology,

a section of the journal

Frontiers in Medicine

Received: 21 October 2021 Accepted: 15 November 2021 Published: 16 December 2021

Citation:

Kamiya K, Shimizu K, Takahashi M, Ando W, Hayakawa H and Shoji N

(2021) Eight-Year Outcomes of Implantation of Posterior Chamber Phakic Intraocular Lens With a Central Port for Moderate to High Ametropia. Front. Med. 8:799078.

doi: 10.3389/fmed.2021.799078

\section{Eight-Year Outcomes of Implantation of Posterior Chamber Phakic Intraocular Lens With a Central Port for Moderate to High Ametropia}

\author{
Kazutaka Kamiya ${ }^{1 *}$, Kimiya Shimizu², Masahide Takahashi², Wakako Ando ${ }^{3}$, \\ Hideki Hayakawa ${ }^{3}$ and Nobuyuki Shoji ${ }^{3}$
}

${ }^{1}$ Visual Physiology, School of Allied Health Sciences, Kitasato University, Tokyo, Japan, ${ }^{2}$ Department of Ophthalmology, Sanno Hospital, Tokyo, Japan, ${ }^{3}$ Department of Ophthalmology, School of Medicine, Kitasato University, Tokyo, Japan

Purpose: To assess the 8-year clinical outcomes of implantation of an implantable collamer lens (ICL) with a central port (KS-Aquaport; EVO-ICL) for moderate to high myopia and myopic astigmatism.

Methods: This retrospective study comprised a total of 177 eyes of 106 patients with spherical equivalents of $-7.99 \pm 3.33 \mathrm{D}$ [mean \pm standard deviation], who underwent EVO-ICL implantation. We evaluated the safety, efficacy, predictability, stability, and adverse events of the surgery, at 1 month, and 1, 2, 4, 6, and 8 years postoperatively.

Results: The logarithm of the minimal angle of resolution (LogMAR) uncorrected distance visual acuity (UDVA) and corrected distance visual acuity (CDVA) were -0.07 \pm 0.17 and $-0.20 \pm 0.09$, respectively, at 8 years postoperatively. The safety and efficacy indices were $1.18 \pm 0.24$ and $0.89 \pm 0.28$, respectively. At 8 years, 83 and $93 \%$ eyes were within $\pm 0.5 \mathrm{D}$ and $\pm 1.0 \mathrm{D}$ of the targeted correction, respectively. Change in manifest refraction from 1 month to 8 years postoperatively was $-0.13 \pm 0.30 \mathrm{D}$. Three eyes (1.7\%) that developed cataracts had a slight pre-existing peripheral anterior subcapsular cataract formation required simultaneous ICL extraction and cataract surgery at 2 or 3 years or ICL size change (1 size up) at 7 years postoperatively. We found that neither significant intraocular pressure (IOP) rise (including pupillary block) nor significant endothelial cell loss occurred in any case throughout the 8-year observation period.

Conclusions: Current ICL implantation with central port technology offered good continuous outcomes for all measures of safety, efficacy, predictability, and stability for correcting moderate to high myopic errors over a long period, thereby suggesting its long-term viability as a surgical approach for the treatment of such eyes.

Keywords: EVO-ICL, phakic IOL, long-term prognosis, safety, efficacy, predictability, stability, myopia 


\section{BACKGROUND}

Implantable Collamer Lens (EVO Visian $\mathrm{ICL}^{\mathrm{TM}}$, STAAR Surgical, Monrovia, CA, USA), a posterior chamber phakic intraocular lens, has been well-recognized as a long-term safe and effective surgery all over the world to correct moderate to high refractive errors (1-6). However, conventional implantable collamer lens (ICL) implantation has several disadvantages over keratorefractive surgeries in the necessity of preoperative laser iridotomy or intraoperative peripheral iridectomy to avoid pupillary block and the possible risk of cataract formation. The EVO ICL with central port technology (KS-AquaPORT V4c and V5; EVO-ICL) was first developed by Shimizu et al. (7, 8) to rectify such drawbacks without significant deterioration in visual performance (9). It has been reported to effectively correct not only moderate to high myopia (10-14) but also low to moderate myopia $(15,16)$. Moreover, ICL surgery is mainly reversible and allows for ICL exchange, unlike laser in situ keratomileusis (LASIK), even when the patients experience unexpected postoperative vision (17). However, to the best of our knowledge, no long-term studies on current ICL implantation with central port technology have so far been conducted spanning more than 7 years. Long-term study on current ICL implantation with central port technology may give us intrinsic insights into understanding the long-term prognosis of the patients undergoing modern ICL surgery, assuming that postoperative complications such as cataract formation and a significant IOP rise could be greatly reduced by introducing this promising technology. The goal of the present study is to retrospectively and longitudinally evaluate the long-term clinical results of EVO-ICL implantation for the correction of moderate to high ametropia, with particular attention to the late-onset complications. As far as we can ascertain, this study assesses the longest-term (up to 8 years) follow-up outcomes in a cohort of patients undergoing current hole technology-based ICL surgery.

\section{MATERIALS AND METHODS}

\section{Study Population}

This study protocol was enrolled with the University Hospital Medical Information Network Clinical Trial Registry (000044268). Our study comprised a total of 156 eyes of 106 consecutive patients, who underwent ICL implantation of the current ICL model (EVO-ICL, V4c and V5) at Kitasato University Hospital between January 2007 and June 2017 for the correction of moderate to high myopia and myopic astigmatism, and who completed a 4-year to 8-year follow-up (visited our hospital at least once during the 4 to 8 years postoperatively for routine examinations). The inclusion criteria for ICL surgery at our institution include unsatisfactory correction with spectacles or contact lenses, age between 20 and 50 years at the time of surgery, stable refraction, -3.00 to -14.0 diopters (D) of myopia with astigmatism of $3 \mathrm{D}$ or less, anterior chamber depth (the corneal endothelium to the crystalline lens, ACD) $\geq 2.8 \mathrm{~mm}$, and endothelial cell density $(\mathrm{ECD}) \geq 1800 \mathrm{cells} / \mathrm{mm}^{2}$. Any history of ocular surgery, corneal diseases, including keratoconus and pellucid marginal degeneration, glaucoma, uveitis, retinal diseases, or other concomitant eye diseases, were excluded from the study. The study was approved by the Institutional Review Board at Kitasato University Hospital (identifier: B21-118) and followed the tenets of the Declaration of Helsinki. Written informed consent for EVO-ICL surgery was obtained from all the patients after explaining the possible consequences.

\section{Outcomes Measures}

Preoperatively and at 1 month, and at 1, 2, 4, 6, and 8 years postoperatively, we assessed the following metrics: the logarithm of the minimal angle of resolution ( $\log$ MAR) of uncorrected distance visual acuity (UDVA) and corrected distance visual acuity (CDVA), the manifest spherical equivalent (MSE), intraocular pressure (IOP) using a non-contact tonometer, ECD using a non-contact specular microscope, and the vault between the anterior surface of the crystalline lens and the posterior surface of the ICL using an anterior segment optical coherence tomography (AS-OCT) (CASIA, Tomey Corporation Co. Ltd., Aichi, Japan) (from 2017 to 2021), in addition to routine slit-lamp biomicroscopic and funduscopic examinations. All available visit data were collected and grouped according to the closest time point. If more than one visit was available within a given grouping, we utilized the visit data comparable to the given time point for this analysis.

\section{Power Calculation and Size Selection}

We determined the ICL size (12.1, 12.6, 13.2, and $13.7 \mathrm{~mm})$, mainly based on the manufacturer's nomogram, using white-towhite distance, and the ACD using a scanning-slit light corneal tomographer (Orbscan,IIz, Bausch\&Lomb, Rochester, USA) or the AS-OCT. We also selected the ICL power using an online calculation and ordering system provided by the manufacturer based on a modified vertex formula $(18,19)$. We usually selected a toric model ICL in eyes with manifest astigmatism of $1 \mathrm{D}$ or more and a non-toric model ICL in eyes with less than $1 \mathrm{D}$.

\section{Surgical Procedures}

We described the details of the surgical procedures in our previous studies $(10-12,15)$. In brief, on the day of the surgery, dilating and topical anesthetic agents were applied. A model V4c or V5 ICL was implanted through a temporal clear corneal incision of 3 to $3.2 \mathrm{~mm}$ after injection of a viscosurgical substance into the anterior chamber. Next, the ICL was inserted into the posterior chamber, the viscosurgical substance was replaced with a balanced salt solution, and a miotic agent was administered. We topically used antibiotic and steroidal medications 4 times daily for 1 week, by gradually reducing the dose.

\section{Statistical Analysis}

The normality of all data samples was first checked using the Shapiro-Wilk test. Since all data fulfilled the criteria for normal distribution, the paired and unpaired $t$-test were used to compare the pre- and post-surgical treatment. The one-way analysis of variance (ANOVA) test was used to assess the time-course of changes, with the Dunnett test being employed for multiple comparisons. The Fisher's exact test was used to compare the percentages between the two groups. Unless otherwise indicated, 
TABLE 1 | Preoperative patient demographics of the study population.

\begin{tabular}{lc}
\hline Parameters & Mean \pm SD (95\% Cl) \\
\hline Number of eyes & 177 \\
Age & $35.9 \pm 7.9$ years (21.2 to 50.6 years) \\
Male: Female & $79: 98$ \\
Log UDVA & $1.32 \pm 0.31(0.72$ to 1.92$)$ \\
LogMAR CDVA & $-0.15 \pm 0.09(-0.34$ to 0.03$)$ \\
Manifest spherical equivalent & $-7.99 \pm 3.33 \mathrm{D}(-14.51$ to $-1.46 \mathrm{D})$ \\
Manifest cylinder & $-1.13 \pm 1.20 \mathrm{D}(-3.48$ to $1.22 \mathrm{D})$ \\
Intraocular pressure & $13.0 \pm 2.5 \mathrm{mmHg}(8.0$ to $17.9 \mathrm{mmHg})$ \\
Mean keratometric readings & $43.51 \pm 1.83 \mathrm{D}(39.92$ to $47.11 \mathrm{D})$ \\
Anterior chamber depth & $3.11 \pm 0.29 \mathrm{~mm}(2.55$ to $3.68 \mathrm{~mm})$ \\
White-to-white distance & $11.6 \pm 0.4 \mathrm{~mm}(10.8$ to $12.3 \mathrm{~mm})$ \\
Central corneal thickness & $528.7 \pm 33.8 \mu \mathrm{m}(462.4$ to $594.9 \mu \mathrm{m})$ \\
Axial length & $26.97 \pm 1.64 \mathrm{~mm}(23.76$ to $30.18 \mathrm{~mm})$ \\
Toric: Non-toric & $76: 101$ \\
ICL size 12.1: $12.6:$ 13.2: 13.7 & $29: 78: 66: 4$
\end{tabular}

$S D$, standard deviation; $C l$, confidence interval; logMAR, logarithm of the minimal angle of resolution; UDVA, uncorrected distance visual acuity; CDVA, corrected distance visual acuity; D, diopter; ICL, implantable collamer lens.

the results are expressed as mean \pm standard deviation [95\% confidence interval (CI)], and a value of $p<0.05$ was considered statistically significant.

\section{RESULTS}

\section{Study Population}

A total of 177 eyes (including 79 of men and 98 of women) of 106 patients met the inclusion criteria of this study. Table 1 shows the preoperative baseline demographics of the study population. The mean follow-up period was $6.3 \pm 1.7$ years $(95 \% \mathrm{CI}, 3.0$ to 9.6 years). The number of eyes examined at each visit were 177 (100\%), 177 (100\%), 177 (100\%), 177 (100\%), 109 (62\%), and $60(34 \%)$, respectively, at 1 month, and 1, 2, 4, 6, and 8 years postoperatively.

\section{Safety and Efficacy Outcomes}

At 4 years postoperatively, 68 eyes (38\%) showed no change in CDVA, 73 eyes (41\%) gained 1 line, and 20 eyes (11\%) gained 2 lines, while 12 eyes (7\%) lost 1 line and 4 eyes (2\%) lost 2 lines. At 6 years postoperatively, 41 eyes (38\%) showed no change in CDVA, 47 eyes (43\%) gained 1 line, and 14 eyes (13\%) gained 2 lines, while 6 eyes (6\%) lost 1 line and 1 eye (1\%) lost 2 lines. At 8 years postoperatively, 22 eyes (37\%) showed no change in CDVA, 22 eyes (37\%) gained 1 line, and 11 eyes (18\%) gained 2 lines, while 5 eyes $(8 \%)$ lost 1 line, but none of the eyes lost 2 lines or more (Figure 1). LogMAR CDVA was $-0.21 \pm 0.08$, $-0.21 \pm 0.08,-0.20 \pm 0.08,-0.21 \pm 0.09,-0.21 \pm 0.09$, and $-0.20 \pm 0.09$, respectively, at 1 month, and $1,2,4,6$, and 8 years postoperatively. We found a significant difference between preoperative CDVA and 4-, 6-, and 8-year postoperative CDVAs ( $p<0.001, p<0.001$, and $p=0.002$, respectively). The safety index (postoperative CDVA/preoperative CDVA) was $1.16 \pm$
$0.24,1.18 \pm 0.26$, and $1.18 \pm 0.24$, respectively, at 4,6 , and 8 years postoperatively.

At 1 month, and $1,2,4,6$, and 8 years postoperatively, $96,86,87,80,83,76$, and $78 \%$ of eyes, and 100, 99, 99, 99, 98, 98, and $100 \%$ of eyes, respectively, had a UDVA of $20 / 20$ and $20 / 40$ or better (Figure 2). LogMAR UDVA was -0.12 $\pm 0.14,-0.12 \pm 0.14,-0.10 \pm 0.14,-0.09 \pm 0.17,-0.08$ \pm 0.21 , and $-0.07 \pm 0.17$, respectively, at 1 month, and 1 , $2,4,6$, and 8 years postoperatively. We found a significant difference between preoperative UDVA and 4-, 6-, and 8year postoperative UDVAs $(p<0.001)$. The efficacy index (postoperative UDVA/preoperative CDVA) was $0.92 \pm 0.29$, $0.90 \pm 0.31$, and $0.89 \pm 0.28$, respectively, at 4,6 , and 8 years postoperatively.

\section{Predictability and Stability Outcomes}

A scatter plot of the attempted vs. the achieved MSE correction is shown in Figure 3. At 1 month, and 1, 2, 4, 6, and 8 years postoperatively, $89,90,96,92,77$, and $83 \%$ of eyes, and 97, 99, 99, 98,90 , and $93 \%$ of eyes were within \pm 0.5 and $1.0 \mathrm{D}$, respectively, of the attempted spherical equivalent correction.

The time-course change in the MSE is shown in Figure 4. Preoperatively, and at 1 month, and 1, 2, 4, 6, and 8 years postoperatively, the MSE was $-7.99 \pm 3.33,-0.21 \pm 0.35,-0.19$ $\pm 0.33,-0.23 \pm 0.42,-0.28 \pm 0.43,-0.33 \pm 0.51$, and -0.28 $\pm 0.36 \mathrm{D}$, respectively (one-way ANOVA, $p<0.001$ ). Changes in MSE refraction from 1 month to 8 years postoperatively were $-0.13 \pm 0.30 \mathrm{D}$.

\section{Intraocular Pressure}

The IOP was $13.0 \pm 2.5,13.4 \pm 2.5,13.3 \pm 2.5,13.6 \pm 2.8,13.5 \pm$ 2.7 , and $14.2 \pm 2.1 \mathrm{mmHg}$, preoperatively, and at $1 \mathrm{month}$, and $1,2,4,6$, and 8 years postoperatively, respectively $(p=0.101)$ (Figure 5). No significant increase in the IOP $(>25 \mathrm{mmHg})$ occurred in any case during the 8-year observation period.

\section{Endothelial Cell Density}

The ECD significantly decreased from $2814 \pm 231$ cells $/ \mathrm{mm}^{2}$ preoperatively to $2672 \pm 237$ cells $/ \mathrm{mm}^{2}$ at 8 years postoperatively $(p<0.001)$. The mean percentage of the ECD loss was $3.6 \pm 7.0$ $\%$ at 8 years postoperatively.

\section{Vault}

The ICL vault was $424 \pm 227,376 \pm 225$, and $347 \pm 181 \mu \mathrm{m}$, at 4,6 , and 8 years postoperatively, respectively $(p=0.250)$. Figure 6 shows the postoperative distribution of the ICL vault. Neither excessive low vault $(<50 \mu \mathrm{m})$ nor excessive-high vault $(>1000 \mu \mathrm{m})$ requiring ICL exchange was found in any case throughout the observation period.

\section{Secondary Surgeries/Adverse Events}

In this series, all surgeries were uneventful, and no apparent intraoperative complications such as an upside-down ICL insertion or traumatic cataract formation occurred. Table 2 summarizes the ICL-related and ICL-unrelated postoperative complications as well as the secondary surgical interventions throughout the 8-year follow-up. Two eyes (1.1\%) required ICL repositioning at 1 week postoperatively (a $90^{\circ}$ rotation to 


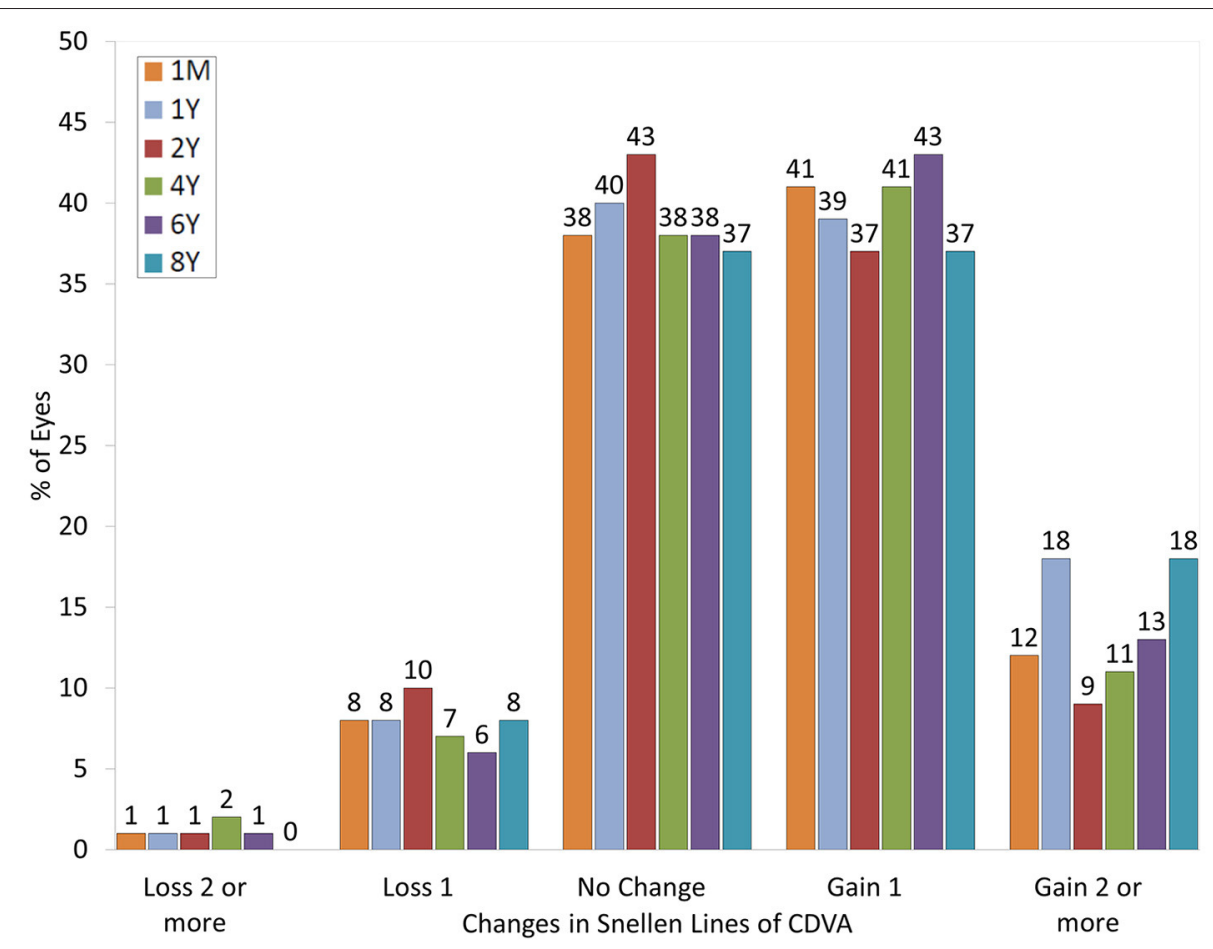

FIGURE 1 | Changes in corrected distance visual acuity (CDVA) after hole implantable collamer lens (EVO-ICL) implantation.

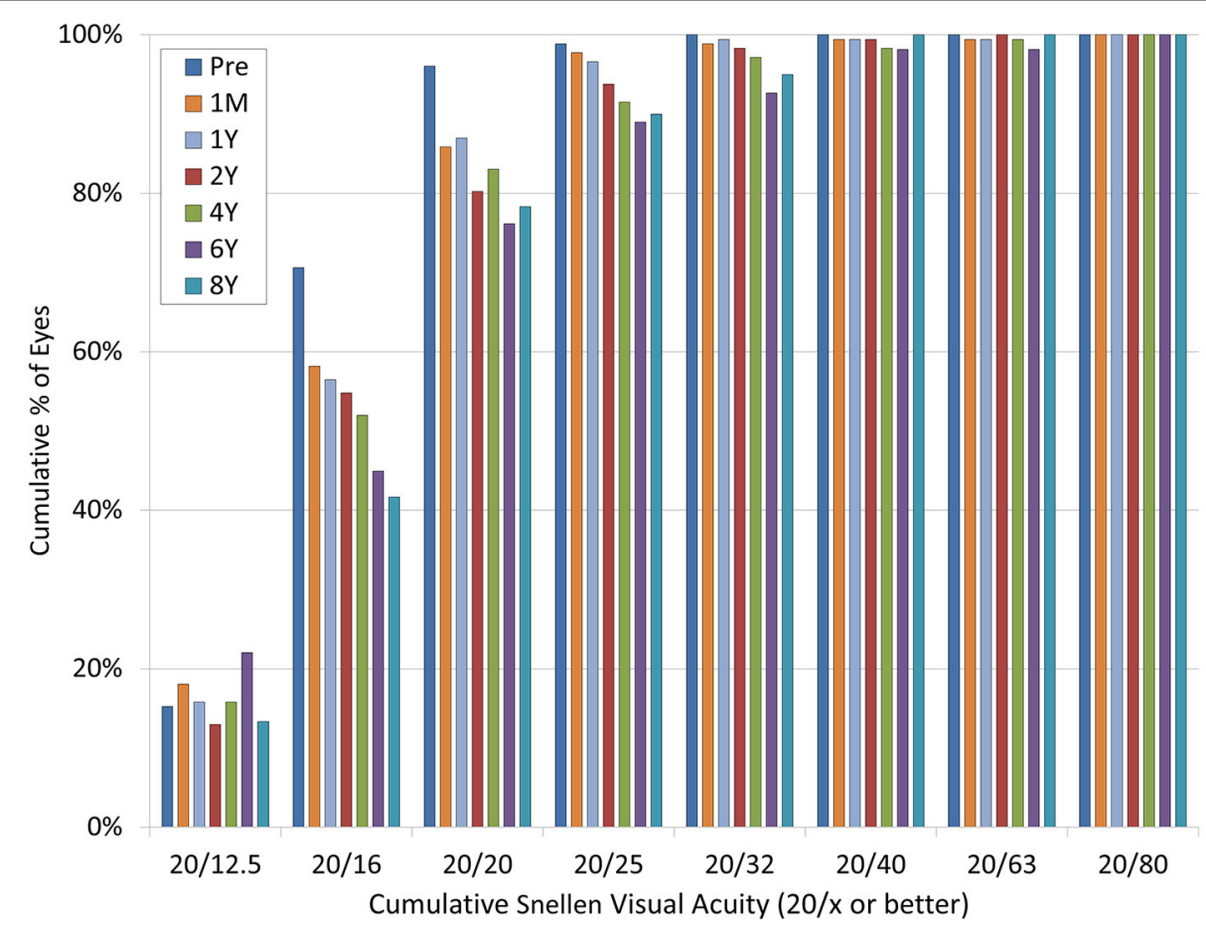

FIGURE 2 | Changes in uncorrected distance visual acuity (UDVA) after hole implantable collamer lens (EVO-ICL) implantation.

vertical orientation due to a high non-toric ICL vault and a realignment due to a toric ICL rotation). Two eyes (1.1\%) required simultaneous ICL extraction and cataract surgery at 2 and 3 years postoperatively due to anterior subcapsular cataract formation. One eye $(0.6 \%)$ required ICL size change ( 1 size up) at 7 years postoperatively to prevent peripheral attachment between the 
ICL and the crystalline lens and the subsequent progression of a peripheral anterior subcapsular cataract. All three eyes (1.7\%) that developed cataracts had a slight pre-existing peripheral anterior subcapsular cataract formation preoperatively, and the

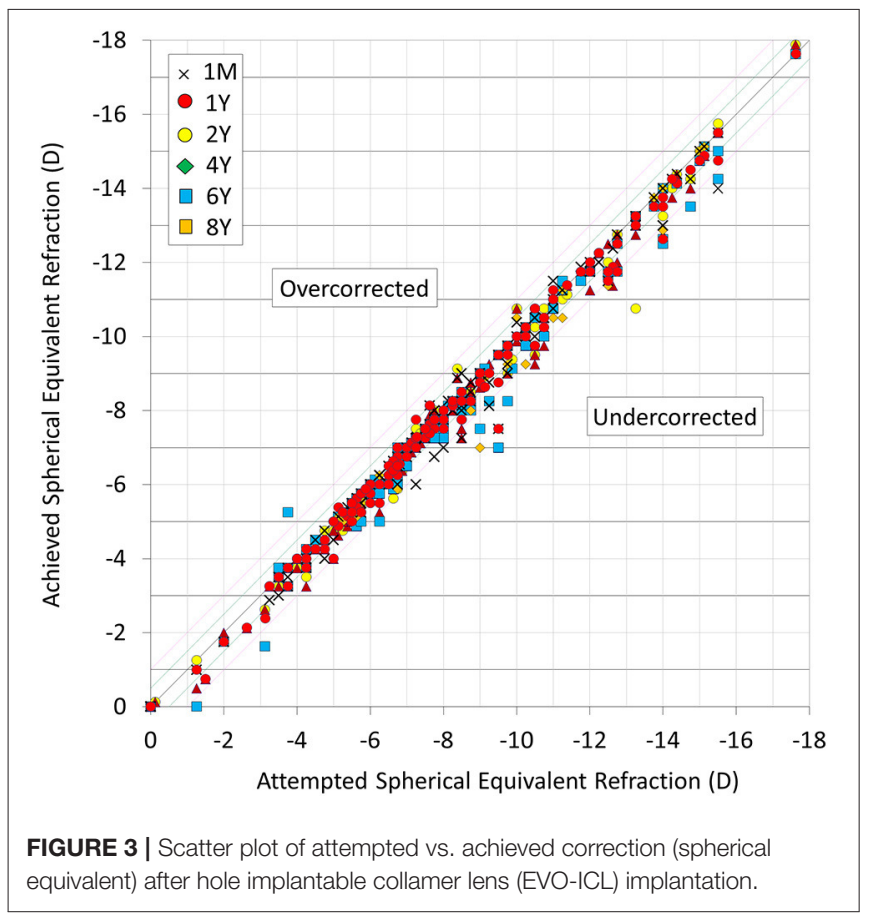

UDVA at the final follow-up was $20 / 20$ or more in these eyes. Two eyes $(1.1 \%)$ required photorefractive keratectomy at 8 years postoperatively due to myopic regression. Otherwise, we found no significant complications, such as substantial IOP rise (>25 mmHg), pigment dispersion glaucoma, pupillary block, consequential ECD loss ( $>20 \%$ ), severe subjective symptoms such as glare or halo, or other vision-threatening complications, during the observation period in this series.

\section{DISCUSSION}

In the present study, our results demonstrated good long-term safety, efficacy, predictability, and stability outcomes during the 8-year observation period. Our results also confirmed that neither a significant IOP rise nor a significant ECD loss occurred in any case. In addition, it was observed that the current EVO-ICL implantation primarily decreased the onset of cataract formation compared to the conventional ICL implantation even at 8 years postoperatively. A total of three eyes (1.7\%) developed cataracts in the current study, but it should be noted that all the eyes had a slight pre-existing peripheral anterior subcapsular cataract formation preoperatively, and the visual prognosis of such eyes was still good. Our findings indicate that this surgical procedure is one of the viable options for correcting moderate to high ametropia over a long period. To the best of our knowledge, this is the longest-term (spanning up to 8 years) study on the clinical outcomes of EVO-ICL implantation. We believe that this information will be clinically helpful for surgeons and patients for understanding the long-term prognosis of the current ICL.

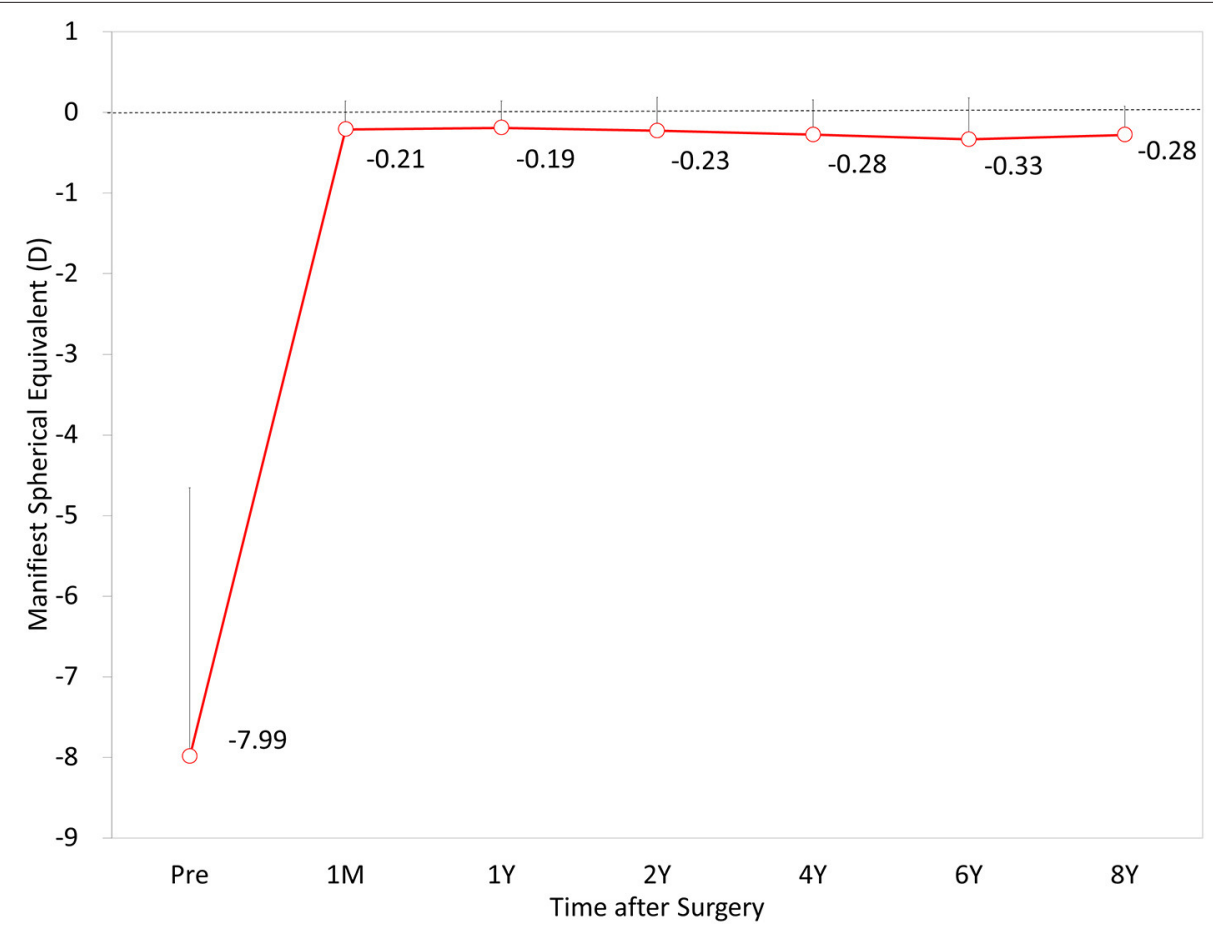

FIGURE 4 | Time course of manifest spherical equivalent (MSE) after hole implantable collamer lens (EVO-ICL) implantation. 


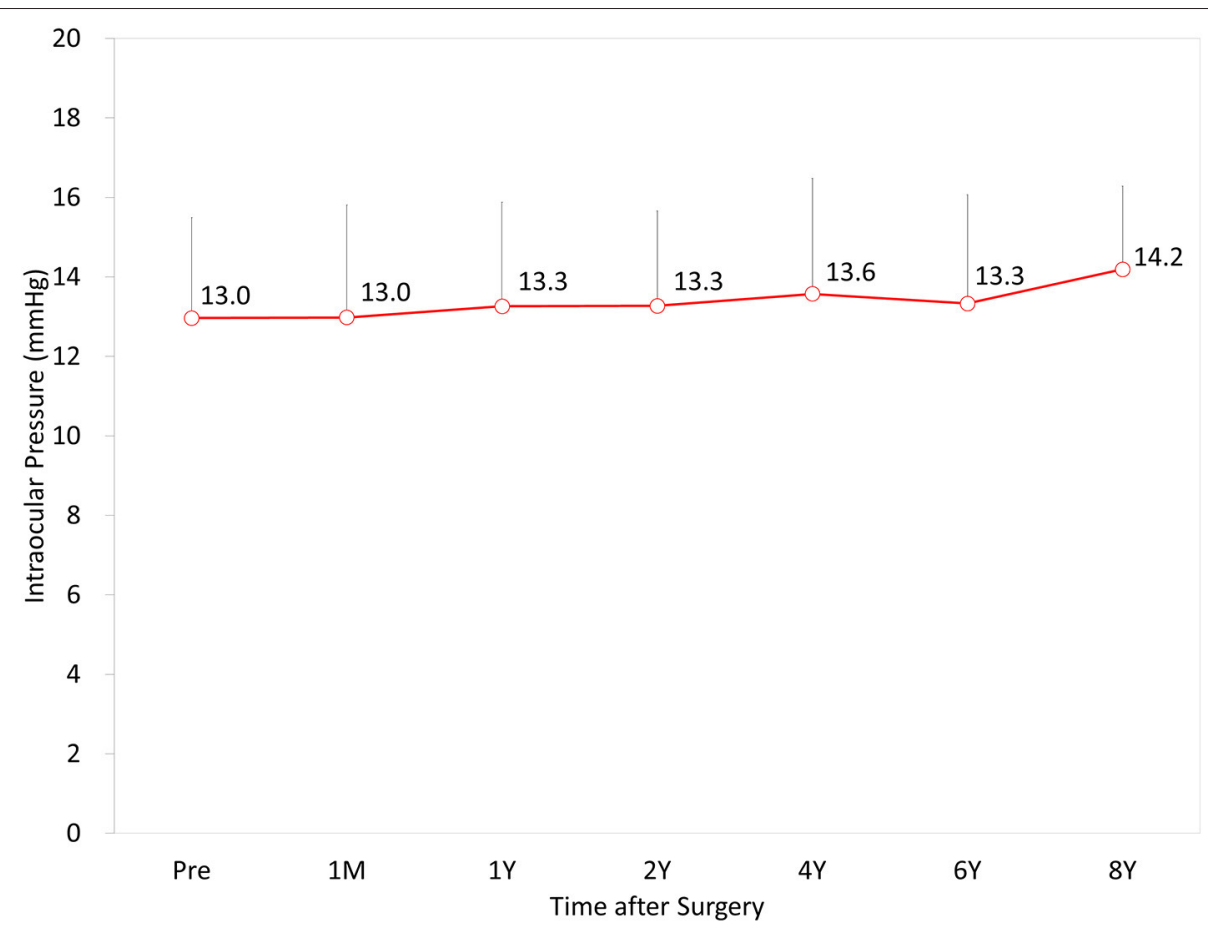

FIGURE 5 | Time course of intraocular pressure (IOP) after hole implantable collamer lens (EVO-ICL) implantation.

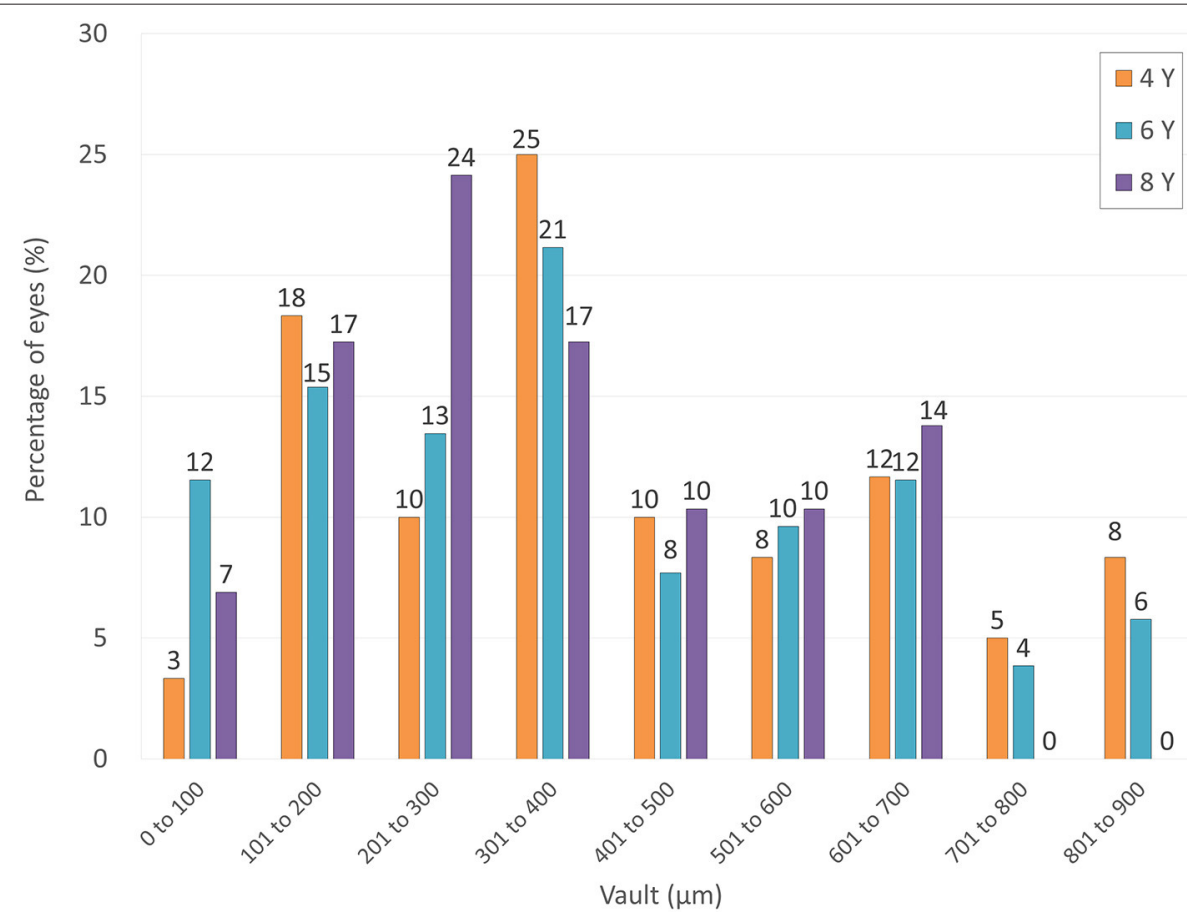

FIGURE 6 | Distribution of eyes according to the vault at 4, 6, and 8 years after hole implantable collamer lens (EVO-ICL) implantation.

Table 3 summarizes the long-term visual and refractive outcomes of conventional ICL implantation without a central port, spanning 10 years or more, and EVO-ICL implantation, spanning 5 years or more, for the myopic study population. Concerning the conventional ICL without a central port, Moya et al. (1) stated that the spherical equivalent refraction was -1.77 
TABLE 2 | Postoperative complications and secondary surgical interventions after hole implantable collamer lens (EVO-ICL) implantation.

\begin{tabular}{|c|c|c|c|c|c|c|c|}
\hline Case & Age (years) & Gender & MSE (D) & ICL type & Onset & Complications & Intervention \\
\hline \multicolumn{8}{|c|}{ ICL-related complications } \\
\hline Case 1 & 29 & Male & -6.00 & Non-toric & 1 week & Excessive high vault & ICL rotation (horizontal to vertical plane) \\
\hline Case 2 & 27 & Female & -5.63 & Toric & 1 week & ICL rotation & ICL repositioning \\
\hline Case 3 & 53 & Female & -6.88 & Non-toric & 2 years & Anterior subcapsular cataract & ICL extraction and cataract surgery \\
\hline Case 4 & 39 & Female & -15.5 & Non-toric & 4 years & Anterior subcapsular cataract & ICL extraction and cataract surgery \\
\hline Case 6 & 35 & Male & -17.63 & Toric & 8 years & Anterior subcapsular cataract & ICL size change ( 1 size up) \\
\hline \multicolumn{8}{|c|}{ ICL-unrelated complications } \\
\hline Case 7 & 44 & Male & -5.13 & Non-toric & 1 years & Retinal tear & Photocoagulation \\
\hline Case 8 & 43 & Male & -6.63 & Toric & 4 years & Epiretinal membrane & Observation \\
\hline
\end{tabular}

MSE, manifest spherical equivalent; D, Diopter; ICL, implantable collamer lens.

TABLE 3 | Summary for long-term outcomes of conventional implantable collamer lens implantation (spanning 10 years or more) and hole implantable collamer lens implantation (5 years or more) for myopia.

\begin{tabular}{|c|c|c|c|c|c|c|c|c|c|c|}
\hline Author & Year & Type & $\begin{array}{l}\text { Period } \\
\text { (years) }\end{array}$ & Eyes & Age (years) & MSE (D) & logMAR UDVA & logMAR CDVA & within $\pm 1.0 \mathrm{D}$ & Cataract \\
\hline Moya et al. (1) & 2015 & ICL V3, V4 (non-hole) & 12 & 110 & $30.69 \pm 5.59$ & $-16.90 \pm 4.26$ & $0.49 \pm 0.25$ & $0.22 \pm 0.25$ & $34.3 \%$ & $13.88 \%$ \\
\hline Kocová et al. (3) & 2017 & ICL V4 (non-hole) & 10 & 40 & $28.28 \pm 6.25$ & $-11.0 \pm 4.45$ & 1.0, decimal & 1.18, decimal & $75.0 \%$ & $12.5 \%$ \\
\hline Nakamura et al. (4) & 2019 & ICL V4 (non-hole) & 10 & 70 & $36.2 \pm 7.7$ & $-9.97 \pm 2.29$ & $-0.01 \pm 0.24$ & $-0.18 \pm 0.07$ & $87.1 \%$ & $10.5 \%$ \\
\hline Alfonso et al. (13) & 2019 & ICL V4c (hole) & 5 & 147 & $31.24 \pm 5.4$ & $-9.20 \pm 3.02$ & $0.02 \pm 0.08$ & $0.02 \pm 0.09$ & $90.1 \%$ & $0 \%$ \\
\hline $\begin{array}{l}\text { Fernández-Vega-Cueto } \\
\text { et al. (14) }\end{array}$ & 2021 & ICL V4c (hole) & 7 & 84 & $31.04 \pm 4.89$ & $-9.35 \pm 2.90$ & $0.17 \pm 0.23$ & $0.02 \pm 0.08$ & $100 \%$ & $0 \%$ \\
\hline Current & 2021 & ICL V4c, V5 (hole) & 8 & 177 & $35.9 \pm 7.9$ & $-7.99 \pm 3.33$ & $-0.07 \pm 0.17$ & $-0.20 \pm 0.09$ & $93 \%$ & $1.7 \%$ \\
\hline
\end{tabular}

ICL, implantable collamer lens; MSE, manifest spherical equivalent; D, diopter; logMAR, logarithm of the minimal angle of resolution; UDVA, uncorrected distance visual acuity; CDVA, corrected distance visual acuity.

$\pm 1.93 \mathrm{D}$ at 12 years postoperatively and that CDVA at the last visit was $0.22 \pm 0.22 \log$ MAR in 144 eyes undergoing ICL surgery. They also found that the incidence of clinically relevant cataracts was $13.88 \%$, which was significantly linked to the use of the V3 model ICL. Guber et al. (2) reported that the rate of lens opacity development was $54.8 \%$ at 10 years postoperatively, owing to the broader view under full dilation and detection of peripheral lens opacity, but there was no significant increase in IOP observed during the 10-year follow-up. Kocová et al. (3) showed that the mean decimal UDVA and CDVA were $1.0 \pm$ 0.37 and $1.18 \pm 0.38$ in myopic eyes, respectively, and $12.5 \%$ of myopic eyes developed cataracts, which significantly affected visual acuity. Nakamura et al. (4) demonstrated that logMAR UDVA and CDVA were $-0.01 \pm 0.24$ and $-0.18 \pm 0.07$ and that 71.4 and $87.1 \%$ of eyes were within 0.5 and $1.0 \mathrm{D}$, respectively, at 10 years postoperatively. They also found that the ECD loss was $5.3 \%$ at 10 years and $10.5 \%$ of 144 eyes developed anterior subcapsular cataracts during the 5- to 10 -year follow-up period.
Choi et al. (5) described no significant changes in the ECD or the IOP at any time point and reported that $12.1 \%$ of eyes developed lens opacities during the 10-year follow-up. We (6) also previously reported that the safety and efficacy indices were respectively $1.13 \pm 0.27$ and $0.83 \pm 0.36$, and that 68.3 and $85.4 \%$ of the eyes were respectively within 0.5 and $1.0 \mathrm{D}$ of the targeted correction at 8 years postoperatively. Concerning the EVO-ICL, there have been only a few studies on the long-term (spanning 5 years or more) outcomes of this new technology-based surgery (12-14). Shimizu et al. (12) first showed, in a pilot study on 32 EVO-ICL-implanted eyes, that logMAR CDVA and UDVA were $-0.24 \pm 0.08$ and $-0.17 \pm 0.14$, respectively, at 5 years postoperatively, and that $96 \%$ of the eyes were within $1.0 \mathrm{D}$ of the targeted correction at 5 years postoperatively. Alfonso et al. (13) described that logMAR CDVA and UDVA were respectively $0.02 \pm 0.08$ and $0.05 \pm 0.11$, at 5 years postoperatively, and that 67.4 and $90.1 \%$ of the eyes were within 0.5 and $1.0 \mathrm{D}$ of the targeted correction, respectively. In a retrospective review of 
84 EVO-ICL-implanted eyes, Fernández-Vega-Cueto et al. (14) recently demonstrated that logMAR CDVA and UDVA were 0.02 \pm 0.08 and $0.17 \pm 0.23$, respectively, at 7 years postoperatively, and 53.57 and $80.95 \%$ of the eyes were within 0.5 and $1.0 \mathrm{D}$ of the targeted correction, respectively. Interestingly, these long-term (spanning 5 years or more) studies confirmed no incidence of cataract formation after EVO-ICL implantation. Packer et al. (20) reviewed a total of 11 publications, including data on 617 eyes with a weighted average follow-up of 13 months, and reported a $0.49 \%$ incidence of asymptomatic anterior subcapsular cataract formation. Although we accept that several background factors, such as patient age, preoperative refraction, type of ICL, surgeon's experience, examiner's skill, and follow-up duration, could play a role in these surgical outcomes, we should be aware that the visual and refractive results were comparable with previous studies on conventional ICL implantation without a central port. However, it should also be noted that the onset of cataract formation has decreased mainly by the introduction of the new technology, possibly due to the improvement of the circulation of the aqueous humor to the anterior surface of the crystalline lens $(7,8)$.

There are several limitations to this study. Firstly, this research was conducted in a retrospective fashion. Indeed, some patients dropped out of the routine follow-up. Considering that visually satisfied patients were lost during the follow-up, we speculate that the actual outcomes could be better than these outcomes. However, this review of the clinical charts may reflect the actual status of hospital-based ICL surgery in a clinical setting. Secondly, the number of patients who completed the 8-year observation were rather limited. A prospective study with a large number of patients would be ideal for accurately grasping the long-term outcomes of this new surgical procedure. Moreover, we did not quantitatively assess the amount of the ICL vault in the early stages of the present study since we introduced the ASOCT in our hospital in 2017. Further research on the stored ICL vault data using the AS-OCT may provide helpful information to assess the long-term changes in the ICL vault in the future.

\section{REFERENCES}

1. Moya T, Javaloy J, Montés-Micó R, Beltrán J, Muñoz G, Montalbán R. Implantable collamer lens for myopia: assessment 12 years after implantation. J Refract Surg. (2015) 31:548-56. doi: 10.3928/1081597X20150727-05

2. Guber I, Mouvet V, Bergin C, Perritaz S, Othenin-Girard P, Majo F. Clinical outcomes and cataract formation rates in eyes 10 years after posterior phakic lens implantation for myopia. JAMA Ophthalmol. (2016) 134:48794. doi: 10.1001/jamaophthalmol.2016.0078

3. Kocová H, Vlková E, Michalcová L, Motyka O. Implantation of posterior chamber phakic intraocular lens for myopia and hyperopia - long-term clinical outcomes. J Fr Ophtalmol. (2017) 40:215-23. doi: 10.1016/j.jfo.2016.10.009

4. Nakamura T, Isogai N, Kojima T, Yoshida Y, Sugiyama Y. Posterior chamber phakic intraocular lens implantation for the correction of myopia and myopic astigmatism: a retrospective 10-year follow-up study. Am J Ophthalmol. (2019) 206:1-10. doi: 10.1016/j.ajo.2019.04.024

5. Choi JH, Lim DH, Nam SW, Yang CM, Chung ES, Chung TY. Tenyear clinical outcomes after implantation of a posterior chamber phakic

\section{CONCLUSIONS}

In summary, our long-term outcomes confirmed that ICL implantation provided good results in terms of long-term safety, efficacy, predictability, and stability, and vision-threatening complications did not occur in any case during the 4- to 8-year observation period. Although the visual and refractive outcomes of EVO-ICL implantation were almost comparable with those of the conventional ICL implantation, we emphasize that late-onset cataract formation, which was one of the concerns about ICL surgery, decreased considerably due to the introduction of the central port technology. Thus, our findings support the view that current ICL implantation with a central port is one of the viable surgical options for correcting moderate to high ametropia over a long period.

\section{DATA AVAILABILITY STATEMENT}

The original contributions presented in the study are included in the article/supplementary material, further inquiries can be directed to the corresponding author/s.

\section{ETHICS STATEMENT}

The studies involving human participants were reviewed and approved by Institutional Review Board at Kitasato University Hospital (identifier: B21-118). Written informed consent for participation was not required for this study in accordance with the national legislation and the institutional requirements.

\section{AUTHOR CONTRIBUTIONS}

$\mathrm{KK}, \mathrm{KS}$, and NS were involved in the design and conduct of the study. MT, WA, and $\mathrm{HH}$ were involved in collection, management, analysis, and interpretation of data. KK, KS, WA, $\mathrm{HH}$, and NS were involved in the preparation, review, and final approval of the manuscript. intraocular lens for myopia. J Cataract Refract Surg. (2019) 45:155561. doi: 10.1016/j.jcrs.2019.06.015

6. Igarashi A, Shimizu K, Kamiya K. Eight-year follow-up of posterior chamber phakic intraocular lens implantation for moderate to high myopia. Am J Ophthalmol. (2014) 157:532-9. doi: 10.1016/j.ajo.2013.11.006

7. Fujisawa K, Shimizu K, Uga S, Suzuki M, Nagano K, Murakami Y, et al. Changes in the crystalline lens resulting from insertion of a phakic IOL(ICL) into the porcine eye. Graefe's Arch Clin Exp Ophthalmol. (2007) 245:11422. doi: 10.1007/s00417-006-0338-y

8. Shiratani T, Shimizu K, Fujisawa K, Uga S, Nagano K, Murakami Y. Crystalline lens changes in porcine eyes with implanted phakic IOL (ICL) with a central hole. Graefes Arch Clin Exp Ophthalmol. (2008) 246:71928. doi: 10.1007/s00417-007-0759-2

9. Uozato H, Shimizu K, Kawamorita T, Ohmoto F. Modulation transfer function of intraocular collamer lens with a central artificial hole. Graefes Arch Clin Exp Ophthalmol. (2011) 249:1081-5. doi: 10.1007/s00417-010-1602-8

10. Shimizu K, Kamiya K, Igarashi A, Shiratani T. Early clinical outcomes of implantation of posterior chamber phakic intraocular lens with a central hole (Hole ICL) for moderate to high myopia. Br J Ophthalmol. (2012) 96:409-12. doi: 10.1136/bjophthalmol-2011-300148 
11. Shimizu K, Kamiya K, Igarashi A, Shiratani T. Intraindividual comparison of visual performance after posterior chamber phakic intraocular lens with and without a central hole implantation for moderate to high myopia. Am J Ophthalmol. (2012) 154:486-94. doi: 10.1016/j.ajo.2012.04.001

12. Shimizu K, Kamiya K, Igarashi A, Kobashi H. Long-term comparison of posterior chamber phakic intraocular lens with and without a central hole (EVO-ICL and conventional ICL) implantation for moderate to high myopia and myopic astigmatism: consort-compliant article. Medicine (Baltimore). (2016) 95:e3270. doi: 10.1097/MD.0000000000003270

13. Alfonso JF, Fernández-Vega-Cueto L, Alfonso-Bartolozzi B, Montés-Micó R, Fernández-Vega L. Five-year follow-up of correction of myopia: posterior chamber phakic intraocular lens with a central port design. J Refract Surg. (2019) 35:169-76. doi: 10.3928/1081597X-20190118-01

14. Fernández-Vega-Cueto L, Alfonso-Bartolozzi B, Lisa C, Madrid-Costa D, Alfonso JF. Seven-year follow-up of posterior chamber phakic intraocular lens with central port design. Eye Vis (Lond). (2021) 8:23. doi: 10.1186/s40662-021-00247-1

15. Kamiya K, Shimizu K, Igarashi A, Kitazawa Y, Kojima T, Nakamura T, et al. Posterior chamber phakic intraocular lens implantation: comparative, multicentre study in 351 eyes with low-to-moderate or high myopia. $\mathrm{Br} \mathrm{J}$ Ophthalmol. (2018) 102:177-81. doi: 10.1136/bjophthalmol-2017-310164

16. Pinto C, Monteiro T, Franqueira N, Faria-Correia F, Mendes J, Vaz F. Posterior chamber collamer phakic intraocular lens implantation: Comparison of efficacy and safety for low and moderate-to-high myopia. Eur J Ophthalmol. (2021) 35:2222-31. doi: 10.1177/112067212110 12861

17. Kamiya K, Shimizu K, Igarashi A, Komatsu M. Comparison of Collamer toric contact lens implantation and wavefront-guided laser in situ keratomileusis for high myopic astigmatism. J Cataract Refract Surg. (2008) 34:1687-93. doi: 10.1016/j.jcrs.2008. 06.030
18. Sanders DR, Vukich JA, Gaston M, Implantable Contact Lens in Treatment of Myopia Study Group. US Food and Drug Administration clinical trial of the Implantable Contact Lens for moderate to high myopia. Ophthalmology. (2003) 110:255-66. doi: 10.1016/S0161-6420(02)01771-2

19. Sanders DR, Doney K, Poco M, ICL in Treatment of Myopia Study Group. United States Food and Drug Administration clinical trial of the Implantable Collamer Lens (ICL) for moderate to high myopia: three-year follow-up. Ophthalmology. (2004) 111:1683-92. doi: 10.1016/j.ophtha.2004.03.026

20. Packer M. The Implantable Collamer Lens with a central port: review of the literature. Clin Ophthalmol. (2018) 12:2427-38. doi: 10.2147/OPTH.S188785

Conflict of Interest: KS was a paid consultant for STAAR Surgical.

The remaining authors declare that the research was conducted in the absence of any commercial or financial relationships that could be construed as a potential conflict of interest.

Publisher's Note: All claims expressed in this article are solely those of the authors and do not necessarily represent those of their affiliated organizations, or those of the publisher, the editors and the reviewers. Any product that may be evaluated in this article, or claim that may be made by its manufacturer, is not guaranteed or endorsed by the publisher.

Copyright (c) 2021 Kamiya, Shimizu, Takahashi, Ando, Hayakawa and Shoji. This is an open-access article distributed under the terms of the Creative Commons Attribution License (CC BY). The use, distribution or reproduction in other forums is permitted, provided the original author(s) and the copyright owner(s) are credited and that the original publication in this journal is cited, in accordance with accepted academic practice. No use, distribution or reproduction is permitted which does not comply with these terms. 